\title{
DOMINATED ESTIMATES OF POSITIVE CONTRACTIONS ${ }^{1}$
}

\author{
R. V. CHACON AND J. OLSEN
}

1. Introduction. In what follows we assume $p$ fixed, $1<p<+\infty$. Let $(X, \mathcal{F}, \mu)$ be a $\sigma$-finite measure space, and let $T$ be a linear operator mapping $L_{p}(X, \mathcal{F}, \mu)$ into $L_{p}(X, \mathcal{F}, \mu)$. If there exists a constant $c>0$ such that

$$
\begin{aligned}
\int \sup _{n} \mid f,(f+T f) / 2 & , \cdots,\left(f+\cdots+T^{n} f\right) / n+\left.1\right|^{p} d \mu \\
& \leqq c^{p} \int|f|^{p} d \mu,
\end{aligned}
$$

for each $f \in L_{p}(X, \mathcal{F}, \mu)$, then we say that $T$ admits of a dominated estimate with constant $c$. If $T$ is a linear operator of norm less than or equal to one, then we say that $T$ is a contraction. If $T$ maps nonnegative functions to nonnegative functions, then we say that $T$ is positive.

Our main result is that positive contractions of $L_{p}(X, \mathcal{F}, \mu)$ having either positive fixed functions or positive semifixed functions (see $\$ 3$ for definitions) admit of a dominated estimate with constant $p /(p-1)$. We then show that this result appears to be behind two theorems seemingly unrelated to each other, one due to E. Stein and the other to A. Ionescu Tulcea.

2. The dominated ergodic theorem. It is now a classical result that if $T$ is at the same time a contraction of $L_{1}(X, \mathcal{F}, \mu)$ and of $L_{\infty}(X, \mathcal{F}, \mu)$ then $T$ admits of a dominated estimate ( $T$ is then also a contraction of $L_{p}(X, \mathcal{F}, \mu)$, by the Riesz convexity theorem, or by a direct calculation). This result is the dominated ergodic theorem and is due to Wiener and Kakutani in less general forms. In the form given here it is due to Hopf and Dunford and Schwartz [2]. We state it as follows:

DOMINATED ERGODIC THEOREM. Let $T$ be a contraction of $L_{1}(X, \mathcal{F}, \mu)$ and of $L_{\infty}(X, \mathcal{F}, \mu)$. Then $T$ admits of a dominated estimate with constant $p /(p-1)$.

Received by the editors September 29, 1967.

1 Research supported in part by NSF Grant GP-3752. 
That the constant can be taken as $p /(p-1)$ follows at once from [2] and a result of [1, Theorem VII-3.4']. (We are indebted to A. Garsia and B. Jamison for this observation.) Although Theorem VII-3.4' is in a section on martingales, its prehistory is ergodic (see [6]).

3. Principal results. Recall that we have assumed $p$ fixed, $1<p<+\infty$, and let $T$ be a positive contraction of $L_{p}(X, \mathcal{F}, \mu)$. We say that $h$ is a positive fixed function of $T$ if $h \in L_{p}(X, \mathcal{F}, \mu), h>0$, and $T h=h$. We say that $h$ is a positive semifixed function of $T$ if $h \in L_{p}(X, \mathcal{F}, \mu), h>0, T h \leqq h$ and $T^{*} h^{p-1} \leqq h^{p-1}$. To justify our terminology we should show that positive fixed functions are also positive semifixed functions. This follows at once from part (iii) of Lemma 3.1.

Lemma 3.1. Let $T$ be a linear operator of $L_{p}(X, \mathcal{F}, \mu)$ and let

$$
h \in L_{p}(X, \mathcal{F}, \mu)
$$

be positive. Let $P$ be the linear operator of $L_{p}(X, \mathcal{F}, m)$ defined by

$$
P f=T(f \cdot h) / h,
$$

where the measure $m$ is obtained by setting $m(A)=\int_{A} h^{p} d \mu$. We have then that

(i) $\|P\| \leqq\|T\|$,

\section{and that}

(ii) $P^{*} g=T^{*}\left(g h^{p-1}\right) / h^{p-1}$.

Furthermore, if $T$ is a positive contraction and $h$ is a positive fixed function, then we have

(iii) $P^{*} 1=1$ (or, equivalently by (ii), $\left.T^{*} h^{p-1}=h^{p-1}\right)$.

Proof. To see that (i) holds, note that

$$
\begin{aligned}
\int|P f|^{p} d m & =\int|T(f h) / h|^{p} h^{p} d \mu \\
& =\int|T(f h)|^{p} d \mu \leqq\|T\|^{p} \int|f h|^{p} d \mu \\
& =\|T\|^{p} \int|f|^{p} d m,
\end{aligned}
$$

and to see that (ii) holds, note that

$$
\int g P(f) d m=\int g[T(f h) / h] h^{p} d \mu=\int T^{*}\left(g h^{p-1}\right) / h^{p-1} f d m .
$$


Finally, to see that (iii) holds, define $\alpha(x)$ as the function such that $P^{*} 1=1+\alpha(x)$, and note that (with $q=p /(p-1)$ )

$$
\int 1 d m=\int 1^{q} d m \geqq \int\left(P^{*} 1\right)^{q} d m=\int(1+\alpha)^{q} d m
$$

since $\left\|P^{*}\right\| \leqq 1$ by (i). Note also that

$$
\int(1+\alpha)^{q} d m \geqq \int 1 d m+q \int \alpha d m,
$$

with strict inequality if $\alpha(x) \neq 0$ on a set of positive measure, since $(1+\alpha(x))^{q}>1+q \alpha(x)$ for each $x$ such that $\alpha(x) \neq 0$. Further, if $h$ is a positive fixed function of $T, P 1=1$ and

$$
\int P^{*}(1) d m=\int 1 \cdot P(1) d m=\int 1 d m=\int 1 d m+\int \alpha d m,
$$

so that $\int \alpha d m=0$. This is incompatible with the previous inequalities unless $\alpha(x) \equiv 0$ and we have that $P^{*} 1=1$.

TheOREM 3.1. Let $T$ be a positive contraction of $L_{p}(X, F, \mu)$ and let $T$ have either a positive fixed function or a positive semifixed function. Then $T$ admits of a dominated estimate with constant $p /(p-1)$.

Proof. It follows from part (iii) of Lemma 3.1 that it is sufficient to prove the theorem under the assumption that $T$ has a positive semifixed function $h$. We again introduce the operator $P$ of $L_{p}(X, \mathcal{F}, m)$ by setting $P f=T(f h) / h$ and $m(A)=\int_{A} h^{p} d \mu$. It follows by part (i) of Lemma 3.1 that $P$ is also a positive contraction of $L_{p}(X, \mathcal{F}, m)$, and it is clear that $P 1 \leqq 1$ and hence that $P$ is a contraction of $L_{\infty}(X, \mathcal{F}, m)$. We have that $P^{*}$ is a positive contraction of $L_{q}(X, \mathcal{F}, m)(q=p /(p-1))$ and by part (ii) of Lemma 3.1 that $P^{*} 1 \leqq 1$ and therefore that $P^{*}$ is a contraction of $L_{\infty}(X, F, m)$. It follows from these remarks and the Riesz convexity theorem that $P$ is a contraction of $L_{1}(X, \mathcal{F}, m)$ and of $L_{\infty}(X, \mathcal{F}, m)$.

It follows from the preceding paragraph that we may apply the dominated ergodic theorem to $P$ to conclude that it admits of a dominated estimate with constant $p /(p-1)$. Further, for every $f \in L_{p}(X, \mathcal{F}$, $\mu)$ we have $f / h \in L_{p}(X, F, m)$, and this, together with the fact that $P^{n} g=T^{n}(g h) / h$ for $g \in L_{p}(X, \mathcal{F}, m)$ enables us to conclude that the dominated estimate for $P$, with respect to the measure $m$, implies the dominated estimate for $T$, with respect to the measure $\mu$ and with the same constant $p /(p-1)$. 
4. Applications. In [4] A. Ionescu Tulcea proved that if $T$ is a positive invertible isometry of $L_{p}(X, \mathcal{F}, \mu)$, then $T$ admits of a dominated estimate with constant $p /(p-1)$. We show that Theorem 3.1 implies the result by means of the following three lemmas. To avoid technical difficulties, we assume that $(X, \mathcal{F}, \mu)$ is a Lebesgue space, although the result is valid in general.

Leмma 4.1. Let $T$ be an isometry of $L_{p}(X, \mathcal{F}, \mu)$ of the form $T f(x)$ $=f(x) r(x)$, where $r(x)$ is a nonnegative measurable function. Then $T=I$.

Proof. Let $A(\alpha, \beta)=\{x: \alpha \leqq r(x) \leqq \beta\}$. Since $T$ is an isometry, we have that $\int_{A(\alpha, \beta)} d \mu=\int_{A(\alpha, \beta)} r^{p} d \mu$, and since

$$
\alpha^{p} \mu(A(\alpha, \beta)) \leqq \int_{A(\alpha, \beta)} r^{p} d \mu \leqq \beta^{p} \mu(A(\alpha, \beta)),
$$

this implies $\alpha \leqq 1 \leqq \beta$ unless $\mu(A(\alpha, \beta))=0$. This is clearly sufficient to establish the lemma.

LеммA 4.2. Let $T$ be an isometry of $L_{p}(X, \mathcal{F}, \mu)$ of the form $T f(x)$ $=f(\tau x) m(x)$, where $m(x)$ is a nonnegative measurable function and $\tau$ is a periodic measurable transformation. Then $T$ is periodic (with the same period).

Proof. Let $n$ be the period of $\tau$ so that $\tau^{n} x=x$ for almost all $x$. Lemma 4.1 implies that $T^{n}=I$, since

$$
T^{n} g(x)=g\left(\tau^{n} x\right) m(x) m(\tau x) \cdots m\left(\tau^{n-1} x\right)=g(x) r(x)
$$

with $r(x)=m(x) m(\tau x) \cdots m\left(\tau^{n-1} x\right)$.

LEMMA 4.3. Let $T$ be a positive invertible isometry of $L_{p}(X, \mathcal{F}, \mu)$. Then $T$ can be approximated in the strong operator topology by periodic positive isometries of $L_{p}(X, \mathcal{F}, \mu)$.

Proof. It is a result essentially due to Banach (see [4]) that positive invertible isometries $T$ can be represented in the form $T f(x)$ $=f(\tau x) r(x)$, where $r$ is the nonnegative measurable function given by $r=[d(\tau \mu) / d \mu]^{1 / p}$, and $\tau$ an invertible measurable transformation. The lemma then follows by Lemma 4.2 from the fact that $\tau$ can be approximated by periodic transformations with respect to the metric $\rho(\tau, \sigma)$ $=\mu\{x: \tau x \neq \sigma x\}$ (by considering the isometries of the form given which these periodic transformations induce). That $\tau$ can be approximated in this way is Linderholm's theorem (see [3, p. 71] for a proof for the measure preserving case easily adaptable to the measurable case). 
The theorem follows at once from Lemma 4.3, since periodic positive isometries have positive fixed functions and consequently admit of a dominated estimate with constant $p /(p-1)$, and so do isometries which they approximate in the strong operator topology. In fact, if $\left\{T_{n}\right\}$ and $T$ are operators of $L_{p}(X, \mathcal{F}, \mu)$ such that $T_{n}$ converges to $T$ in the strong operator topology, and such that each $T_{n}$ admits of a dominated estimate with constant $c$, then $T$ admits of a dominated estimate with constant $c$ as well.

In [5] E. M. Stein proved that if $P$ is a positive selfadjoint contraction of $L_{2}(X, \mathcal{F}, \mu)$, then $\left\|\sup _{n} P^{n} f(x)\right\| \leqq 5\|f(x)\|$. The key steps in the proof consist of proving that $P$ admits of a dominated estimate, and then of reducing this estimate for averages to the desired estimate. We prove three lemmas which show that positive selfadjoint contractions of $L_{2}(X, \mathcal{F}, \mu)$ admit of a dominated estimate with constant 2, from which the second part of Stein's proof yields $\left\|\sup _{n} P^{n} f(x)\right\|$ $\leqq 3\|f(x)\|$. We assume $\mu(X)<+\infty$ without loss of generality (the $\sigma$-finite case follows from this by considering operators of the form $\chi_{A} T \chi_{A}$ where $\mu(A)<+\infty$ and $\chi_{A}$ is the characteristic function of $A)$.

LemMA 4.4. Let $T$ be a positive selfadjoint contraction of $L_{2}\left(X, \mathcal{F}_{n}, \mu\right)$, where $\mathcal{F}_{n}$ is a finite field. Then $T$ admits of a dominated estimate with constant 2 .

Proof. Note that the positive selfadjoint contractions of $L_{2}\left(X, F_{n}, \mu\right)$ can be represented by nonnegative matrices and that those with strictly positive elements are dense. It suffices therefore to prove the lemma under this assumption. An application of the Frobenius theorem yields that $T$ has a positive eigenvalue $\lambda$ with an associated positive eigenfunction $h$. Since $T$ is a contraction $\lambda \leqq 1$ and we have $T h \leqq h$. Further, since $T$ is selfadjoint, $T^{*} h \leqq h$ and we have that $h$ is a positive semifixed function of $T$ and therefore Theorem 3.1 implies that $T$ admits of a dominated estimate with constant 2 .

LEMмA 4.5. Let $T$ be a positive selfadjoint contraction of $L_{2}(X, \mathcal{F}, \mu)$ and let $\mathcal{F}_{n}$ be a finite subfield of $\mathcal{F}$. Then the operator $E_{n} T E_{n}$ admits of a dominated estimate with constant 2 , where $E_{n} f=E\left\{f \mid \mathcal{F}_{n}\right\}$.

Proof. Since $E_{n}$ is a positive selfadjoint contraction, so is $E_{n} T E_{n}$. We easily see that $E_{n} T E_{n}$ maps $L_{2}\left(X, \mathcal{F}_{n}, \mu\right)$ into $L_{2}\left(X, \mathcal{F}_{n}, \mu\right)$, and therefore Lemma 4.4 implies that $E_{n} T E_{n}$ so restricted admits of a dominated estimate with constant 2 . This is sufficient to prove the lemma since $E_{n}$ is a contraction. 
Lemma 4.6. Let $T$ be a bounded operator of $L_{2}(X, F, \mu)$ into $L_{2}(X, F, \mu)$ and let $\left\{\mathcal{F}_{n}\right\}$ be a sequence of finite fields converging monotonically to $\mathscr{F}$. Then $E_{n} T E_{n}$ converges to $T$ in the strong operator topology, where $E_{n} f=E\left\{f \mid \digamma_{n}\right\}$.

PRoof. Follows trivially from the martingale convergence theorem [1, Theorem VII-4.1].

\section{BIBLIOGRAPHY}

1. J. L. Doob, Stochastic processes, Wiley, New York, 1953.

2. N. Dunford and J. T. Schwartz, Convergence almost everywhere of operator averages, J. Math. Mech. 5 (1956), 129-178.

3. P. R. Halmos, Lectures on ergodic theory, The Mathematical Society of Japan, Tokyo, 1956.

4. A. Ionescu Tulcea, Ergodic properties of isometries in $L_{p}$ spaces, $1<p<\infty$, Bull. Amer. Math. Soc. 70 (1964), 366-371.

5. E. M. Stein, On the maximal ergodic theorem, Proc. Nat. Acad. Sci. U.S.A. 47 (1961), 1894-1897.

6. N. Wiener, The ergodic theorem, Duke Math. J. 5 (1939), 1-18.

UNIVERSITY OF MINNESOTA 